\title{
ON A NONTRIVIAL HIGHER EXTENSION OF REPRESENTABLE ABELIAN SHEAVES ${ }^{1}$
}

\author{
BY LAWRENCE S. BREEN
}

Communicated May 2, 1969

Let $S$ be the category of abelian sheaves in the fppf topology over a base scheme $S$, as defined in Demazure and Grothendieck [3, exposé IV §6.3]. This is an abelian category with enough injectives (see Artin $[1,1.6,1.8])$. For any $F$ in $\delta$ and any integer $i \geqq 0$, the functor $\operatorname{Ext}^{i}(F,-)$ from $S$ to the category of abelian groups is defined in the usual manner to be the $i$ th derived functor of the functor $\operatorname{Hom}(F,-)$. Let $S=\operatorname{Spec}(k)$ where $k$ is a separably closed field of characteristic 2; we denote by $\alpha_{2}$ the scheme Spec $\left(k[x] /\left(x^{2}\right)\right)$ with the usual group law (see for example Oort [8]), by $G_{m}$ the multiplicative group scheme, and identify these objects of the category $\mathfrak{e}$ of commutative algebraic group schemes over $S$ with the objects in $S$ which they represent. We show that $\operatorname{Ext}^{2}\left(\alpha_{2}, G_{m}\right) \neq 0$.

Via the identification just mentioned, $\mathfrak{e}$ is a full subcategory of $\mathcal{S}$ which however does not contain enough injectives. It is nonetheless possible to define a functor $\mathrm{Ext}^{i}$ within the category $\mathfrak{e}$. For $G, G^{\prime} \in \mathcal{C}$, define $\operatorname{Ext}^{i}\left(G, G^{\prime}\right)$ to be the group of equivalence classes of $i$-fold Yoneda extensions in $\mathcal{e}$ of $G$ by $G^{\prime}$. This point of view, which was introduced by Serre in [9], was systematically developed by Oort in [8]. Oort shows in particular that $\operatorname{Ext}^{i}\left(H, G_{m}\right)=0$ for $i \geqq 1$, where $H$ is any finite group scheme over an algebraically closed groundfield. Our computation thus illustrates the fact that the two definitions of Ext $^{i}$ are not equivalent.

I wish to thank A. K. Bousfield and B. Mazur for their valuable help during the preparation of this work.

The technique used below in computing $\operatorname{Ext}^{i}\left(\alpha_{2}, G_{m}\right)$ (where henceforth we will always mean the first definition of $\left.\mathrm{Ext}^{i}\right)$ is that of [2]; since only a small part of the theory described there is needed in our special case, we restate in detail the facts required.

Eilenberg and MacLane have defined [4, p. 659], [5] for every abelian group $G$ a complex of free abelian groups $A(G)$ called the abelian complex of $G$ :

1 This work was supported by NSF grant GP 9152. 


$$
\begin{aligned}
& A(G)_{i}=0 \quad i \leqq 0 \\
& A(G)_{1}=Z[G] \text { is generated by elements }[x] \\
& A(G)_{2}=Z\left[G^{2}\right] \text { is generated by elements }[x \mid y] \\
& A(G)_{3}=Z\left[G^{3}\right] \times Z\left[G^{2}\right] \text { is generated by elements }[x|y| z],\left[\left.x\right|_{2} y\right] \\
& A(G)_{4}=Z\left[G^{4}\right] \times Z\left[G^{3}\right] \times Z\left[G^{3}\right] \times Z\left[G^{2}\right]
\end{aligned}
$$

is generated by elements $[x|y| z \mid w],\left[x|y|_{2} z\right],\left[\left.x\right|_{2} y \mid z\right],\left[\left.x\right|_{3} y\right]$

where in each case $x, y, z, w$ range over all elements of $G$. The boundaries $\partial_{i}: A(G)_{i} \rightarrow A(G)_{i-1}$ are defined on the generators and extended by linearity:

$$
\begin{aligned}
\partial_{1} & =0 \\
\partial_{2}[x \mid y] & =[x]-[x+y]+[y] \\
\partial_{3}[x|y| z] & =[y \mid z]-[x+y \mid z]+[x \mid y+z]-[x \mid y], \partial_{3}\left[\left.x\right|_{2} y\right]=[x \mid y]-[y \mid x] \\
\partial_{4}[x|y| z \mid w] & =[y|z| w]-[x+y|z| w]+[x|y+z| w]-[x|y| z+w]+[x|y| z] \\
\partial_{4}\left[x|y|_{2}\right] & =\left[\left.x\right|_{2} z\right]-\left[x+\left.y\right|_{2} z\right]+\left[\left.y\right|_{2} z\right]+[x|y| z]-[x|z| y]+[z|x| y] \\
\partial_{4}\left[\left.x\right|_{2} y \mid z\right] & =\left[\left.x\right|_{2} y\right]-\left[\left.x\right|_{2} y+z\right]+\left[\left.x\right|_{2} z\right]-[x|y| z]+[y|x| z]-[y|z| x] \\
\partial_{4}\left[\left.x\right|_{8} y\right] & =-\left[\left.x\right|_{2} y\right]-\left[\left.y\right|_{2} x\right] .
\end{aligned}
$$

The homology of this complex (which is just the stable homology of the Eilenberg-MacLane space) was computed in low dimensions by Eilenberg and MacLane [5] and in the general case by Cartan. In the lowest dimensions one has:

$$
\begin{gathered}
\sigma: G \rightarrow H_{1}(A(C)) \quad \sigma(x)=[x] \\
H_{2}(A(G))=0 \\
\gamma: G / 2 G \rightarrow H_{3}(A(G)) ;
\end{gathered}
$$

$\gamma$ is defined on a representative $x \in G$ of $G / 2 G$ by $\gamma(x)=\left[\left.x\right|_{2} x\right]$. One checks that $\gamma(2 x)$ is a boundary, so $\gamma$ passes to the quotient.

The complex $A(G)$ and the isomorphisms $\sigma$ and $\gamma$ are functorial in $G$, so one can define without difficulty, for any abelian presheaf $P$, a complex of abelian presheaves $A(P)$ with again $\sigma: P \rightarrow H_{1}(A(P)$ ), $H_{2}(A(P))=0, \gamma: P / 2 P \rightarrow H_{3}(A(P))$. Applying the functor "associated sheaf," which is exact $[1,1.6]$, one defines for any abelian sheaf $F$ on some topology a complex of abelian sheaves $A(F)$ of the form $A(F)_{1}$ $=\boldsymbol{Z}[F], A(F)_{2}=\boldsymbol{Z}\left[F^{2}\right]$, etc. . . , with $\sigma: F \rightarrow H_{1}(A(F)), H_{2}(A(F))$ $=0, \gamma: F / 2 F \rightarrow H_{3}(A(F))$ where $F / 2 F$ is the cokernel of multiplication by 2 in the category of abelian sheaves (for more details, see [2]). 
Examine now the special case where $F=\alpha_{2}$ (considered as an fppf sheaf over Spec $(k)$ ). In this case the complex $A(F)$ is $G_{m}$-acyclic: $\operatorname{Ext}^{i}\left(Z\left[\alpha_{2}\right], G_{m}\right)=H_{f p p j}^{i}\left(\alpha_{2}, G_{m}\right)$ since both are the value at $G_{m}$ of the $i$ th derived functor of the functor $\operatorname{Hom}\left(Z\left[\alpha_{2}\right],-\right)=H_{\text {fppf }}^{0}\left(\alpha_{2},-\right)$. Now, since $G_{m}$ is smooth, $H_{f p p s}^{i}\left(\alpha_{2}, G_{m}\right)=H_{e l}^{i}\left(\alpha_{2}, G_{m}\right)$ (see [7, Appen$\mathrm{dix}]$ ) and this last group is trivial for $i$ positive since the affine ring of $\alpha_{2}$ is Henselian (in fact Artinian) with separably closed residue class field $[1,4.9]$.

In any abelian category with enough injectives, there is a wellknown spectral sequence comparing the homology and the cohomology of an acyclic complex [6, p. 100]:

$$
E_{2}^{p, q}=\operatorname{Ext}^{p}\left(H_{q}\left(A\left(\alpha_{2}\right), G_{m}\right) \Rightarrow H^{p+q}\left(A\left(\alpha_{2}\right) ; G_{m}\right)\right.
$$

where $H^{j}\left(A\left(\alpha_{2}\right) ; G_{m}\right)$ is the $j$ th cohomology group of the complex $A^{*}\left(\alpha_{2}, G_{m}\right)$ with $A^{q}\left(\alpha_{2}, G_{m}\right)=\operatorname{Hom}\left(A\left(\alpha_{2}\right)_{q}, G_{m}\right)$ for all integers $q$, the coboundary maps being the obvious ones.

$E_{2}^{0.3}=\operatorname{Hom}\left(F / 2 F, G_{m}\right)=\operatorname{Hom}\left(\alpha_{2}, G_{m}\right)$ since multiplication by 2 is trivial on $\alpha_{2}$. This last group is trivial as is well known (and can be checked easily). Since $H_{q}\left(A\left(\alpha_{2}\right)\right)=0$ for $q \leqq 0$ and $q=2, E_{2}^{p, q}=0$ for those values of $q$. The spectral sequence thus degenerates in low dimensions. In particular

$$
E_{2}^{2,1}=\operatorname{Ext}^{2}\left(\alpha_{2}, G_{m}\right) \approx H^{3}\left(A\left(\alpha_{2}\right) ; G_{m}\right) .
$$

We will exhibit a 3-cocycle which is not a coboundary. To simplify the notation we will, in all the following, write $k\left[x_{1}, \cdots, x_{n}\right]^{*}$ for the group of invertible elements in the ring

$$
k\left[x_{1}, \cdots, x_{n}\right] /\left(x_{1}^{2}, \cdots, x_{n}^{2}\right)
$$

(i.e. the group of truncated polynomials with nontrivial constant terms). A 3-cochain is an element of

$$
\begin{aligned}
\operatorname{Hom}\left(A\left(\alpha_{2}\right)_{3}, G_{m}\right) & =\operatorname{Hom}\left(Z\left[\alpha_{2}^{3}\right] \times Z\left[\alpha_{2}^{2}\right], G_{m}\right) \\
& =\operatorname{Hom}\left(Z\left[\alpha_{2}^{8}\right], G_{m}\right) \oplus \operatorname{Hom}\left(Z\left[\alpha_{2}^{2}\right], G_{m}\right) \\
& =H^{0}\left(\alpha_{2}^{8}, G_{m}^{-}\right) \oplus H^{0}\left(\alpha_{2}^{2}, G_{m}\right) \\
& =k[x, y, z]^{*} \oplus k[x, y]^{*} .
\end{aligned}
$$


A 3-cochain is thus a pair $(f, g)$ with $f=f(x, y, z) \in k[x, y, z]^{*}, g$ $=g(x, y) \in k[x, y]^{*}$. The cocycle condition for such a pair is $\partial(f, g)$ $=(1,1,1,1)$ where

$$
\begin{aligned}
\partial(f, g)= & \left(f_{1}, f_{2}, f_{3}, f_{4}\right) \in k[x, y, z, w]^{*} \oplus k[x, y, z]^{*} \oplus k[x, y, z]^{*} \\
& \oplus k[x, y]^{*}
\end{aligned}
$$

with

$$
\begin{aligned}
f_{1}(x, y, z, w) & =f(y, z, w) f(x+y, z, w)^{-1} f(x, y+z, w) f(x, y, z+w)^{-1} f(x, y, z), \\
f_{2}(x, y, z) & =g(x, z) g(x+y, z)^{-1} g(y, z) f(x, y, z) f(x, z, y)^{-1} f(z, x, y), \\
f_{3}(x, y, z) & =g(x, y) g(x, y+z)^{-1} g(x, z) f(x, y, z)^{-1} f(y, x, z) f(y, z, x)^{-1}, \\
f_{4}(x, y) & =g(x, y)^{-1} g(y, x)^{-1} .
\end{aligned}
$$

Similarly, $(f, g)$ is a coboundary if there exists an element $h=h(x, y)$ $\in k[x, y]^{*}$ such that

$$
\begin{aligned}
f(x, y, z) & =h(y, z) h(x+y, z)^{-1} h(x, y+z) h(x, y)^{-1}, \\
g(x, y) & =h(x, y) h(y, x)^{-1} .
\end{aligned}
$$

Consider the 3-cochain $(1,1+u x y) \in k[x, y, z]^{*} \oplus k[x, y]^{*}$, where $u$ is any nontrivial element of $k$. One checks without difficulty that this pair satisfies the cocycle condition above and is not a coboundary.

REMARKs. 1. It is immediate that if $u$ and $v$ are distinct elements of $k$, the pairs $(1,1+u x y)$ and $(1,1+v x y)$ are not cohomologous, so $k$ (with the additive group law) is a subgroup of $\operatorname{Ext}^{2}\left(\alpha_{2}, G_{m}\right)$. In fact one can prove that $k \approx \operatorname{Ext}^{2}\left(\alpha_{2}, G_{m}\right)$.

2. More generally, if $k$ is any separably closed field of characteristic $p \neq 0, \operatorname{Ext}^{i}\left(\alpha_{p}, G_{m}\right)=0$ for $0<i<2 p-2, \operatorname{Ext}^{2 p-2}\left(\alpha_{p}, G_{m}\right) \approx k$.

3. Let $A$ be an abelian variety with $\operatorname{Hom}\left(\alpha_{2}, A\right) \neq 0$. One concludes that $\operatorname{Ext}^{3}\left(A, G_{m}\right) \neq 0$.

4. It is possible to give an explicit description of these nontrivial extensions as Yoneda extensions in $\mathcal{S}$ (involving of course some nonrepresentable sheaves).

5. The cocyle exhibited above also gives a nontrivial element of $\operatorname{Ext}^{2}\left(\alpha_{2}, G_{m}\right)$ where we now identify the commutative group schemes with the objects in the category of abelian presheaves on $\operatorname{Spec}(k)$ which they represent and mean by Ext ${ }^{2}$ the second derived functor of Hom in this category.

\section{REFERENCES}

1. M. Artin, Grothendieck topologies, Mimeographed notes, Harvard University, Cambridge, Mass., 1962. 
2. L. Breen, Extensions of abelian sheaves and Eilenberg-MacLane algebras, Thesis, Columbia University, New York, N. Y., 1969.

3. M. Demazure and A. Grothendieck, Schémas en groupes, Mimeographed notes, Séminaire de Géométrie Algébrique, Inst. Hautes Études Sci., Paris, 1963.

4. S. Eilenberg and S. MacLane, Cohomology theory of Abelian groups and homotopy theory. II, Proc. Nat. Acad. Sci. U.S.A. 36 (1950), 657-663.

5. - On the groups $H(\mathrm{II}, n)$. I, II, Ann. of Math. (2) 58 (1953), 55-106; (2) 60 (1954), 49-139.

6. R. Godement, Topologie algêbrique et thêorie des faisceaux, Actualités Sci. Indust., no. 1252, Hermann, Paris, 1964.

7. A. Grothendieck, Le groupe de Brauer. III, Mimeographed notes, Reprint., North-Holland, Amsterdam, 1969.

8. F. Oort, Commutative group schemes, Lecture Notes in Math. no. 15, SpringerVerlag, Berlin, 1966.

9. J.-P. Serre, Groupes algébriques et corps de classes, Actualités Sci. Indust., no. 1264, Hermann, Paris, 1959.

Massachusetts Institute of Technology, Cambridge, Massachusetts 02139 